\title{
Asymptotic symmetries and electromagnetic memory
}

\author{
Sabrina Pasterski \\ Center for the Fundamental Laws of Nature, Harvard University, \\ 17 Oxford Street, Cambridge, MA, 02138 U.S.A. \\ E-mail: spasterski@fas.harvard.edu
}

ABSTRACT: Recent investigations into asymptotic symmetries of gauge theory and gravity have illuminated connections between gauge field zero-mode sectors, the corresponding soft factors, and their classically observable counterparts - so called "memories". Namely, low frequency emissions in momentum space correspond to long time integrations of the corresponding radiation in position space. Memory effect observables constructed in this manner are non-vanishing in typical scattering processes, which has implications for the asymptotic symmetry group. Here we complete this triad for the case of large U(1) gauge symmetries at null infinity. In particular, we show that the previously studied electromagnetic memory effect, whereby the passage of electromagnetic radiation produces a net velocity kick for test charges in a distant detector, is the position space observable corresponding to the Weinberg soft photon pole in momentum space scattering amplitudes.

Keywords: Classical Theories of Gravity, Gauge Symmetry, Space-Time Symmetries

ARXIV EPRINT: 1505.00716 


\section{Contents}

1 Introduction 1

2 Conventions 3

3 Maxwell in the radiation zone 4

3.1 Boundary conditions 4

3.2 Electromagnetic memory 5

$\begin{array}{lll}3.3 & \text { Weinberg soft factor } & 7\end{array}$

3.4 Large U(1) symmetry 9

4 Measurement 11

5 Discussion 12

\section{Introduction}

Recent investigations into asymptotic symmetries of gauge theory and gravity have illuminated connections between gauge field zero-mode sectors, the corresponding soft factors, and their classically observable counterparts called "memories". The connections between these concepts can be illustrated by the following triangle:

THE

TRIAD i) Weinberg - photon $\mathcal{O}\left(\frac{1}{\omega}\right)$

ii) Weinberg - graviton $\mathcal{O}\left(\frac{1}{\omega}\right)$

iii) Cachazo \& Strominger - graviton $\mathcal{O}(1)$

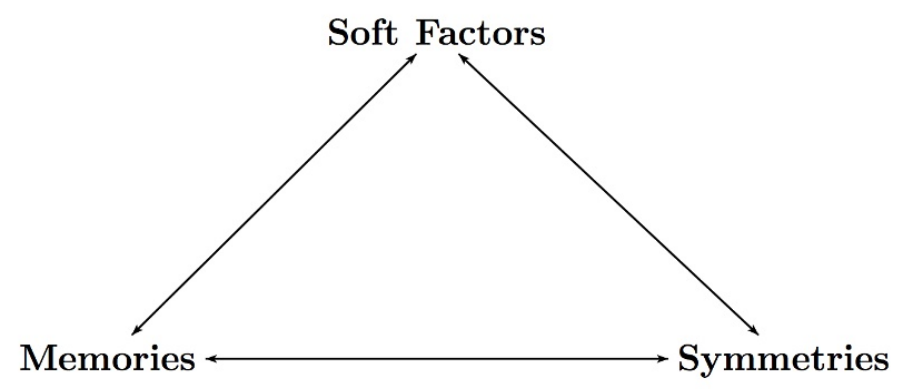
i) Liénard-Wiechert / Bieri \& Garfinkle
ii) Zeldovich \& Polnarev / Christodoulou
i) e-charge large U(1)
iii) Pasterski, Strominger, \& Zhiboedov
ii) $p^{\mu} \quad$ supertranslations
iii) $J^{\mu \nu} \quad$ superrotations

(global)

(asymptotic)

Recent literature has drawn the links connecting soft factors, symmetries, and memories for two of the three sets above (see [1] for ii), [2] for iii)). This paper closes the 
triangle for case i). Meanwhile, the individual vertices and edges have been studied for quite some time. Of the connections/edges, the oldest and most well known are those that lie between the leading gauge and gravity soft factors and their corresponding global symmetries: charge and four-momentum conservation, respectively, as derived by Weinberg [3]. Also in the 1960's, Bondi, van der Burg, Metzner, and Sachs (BMS) worked out the symmetry group for asymptotically flat spacetimes [4, 5]. However, it was not until [6] that these two lines of research were connected, with Weinberg's soft graviton theorem reinterpreted as a Ward identity for the BMS asymptotic symmetry group [7]. The final step of connecting these soft-factors/asymptotic symmetries to a classical observable came in [1], which found that Weingberg's soft graviton theorem corresponds to the gravitational memory effect [8-10].

The utility of thinking in terms of the above triangle is best illustrated in cases where some of the vertices are 'missing,' and the above connections can serve as a guide to fill in these gaps. The superrotation iteration iii) is a prime example. In the early 2000's, it was suggested [11] that the globally defined BMS supertranslations could be accompanied by locally defined superrotations, extending the standard homogenous Lorentz group [12-14]. The utility of such an extension was demonstrated by Strominger and collaborators when they derived the corresponding tree-level subleading soft factor [15], showed its connection to superrotation generators [16], and completed the above triangle by proposing the spin memory effect [2].

The electromagnetic iteration is one where the vertices are known separately but only some of the connections have been made. The first step, linking soft factors and symmetries was made in [17], connecting the leading Weinberg soft photon factor to an asymptotic large $\mathrm{U}(1)$ gauge symmetry. What remains is to draw the final links to the electromagnetic version of a "memory effect". We are aided by recent work discussing the electromagnetic analog of gravitational memory [18]. Intent on establishing a connection between electromagnetic memory, the asymptotic U(1) gauge symmetry of [17] and the leading Weinberg soft factor, the goal of this paper is to show that a measurement of the electromagnetic memory produced by scattering charges with fixed initial and final momenta yields the soft factor corresponding to the $\mathcal{S}$-matrix element with those in and out states plus an additional soft photon.

This paper is organized as follows. In section 2 we introduce conventions, and set the groundwork for the finite- $r$ measurement interpretation. Section 3 describes different manifestations of the electromagnetic memory effect related to the massive/massless splitting of [18]. In 3.1, we outline the applicable boundary conditions. In 3.2, we clarify what one means by a "memory" effect and discuss equations relevant to the results of [18]. Section 3.3 explores the connection to Weinberg's soft factor in the massive case, as can be seen from using retarded radiation solutions in classical electromagnetism a la [19]. Then in 3.4, we review the asymptotic U(1) gauge symmetry of [17] and how the previous discussions connect to the new boundary conditions for a massless scattering process. Section 4 describes an alternative measurement for the electromagnetic memory effect, where suspension of test charges in a viscous fluid results in a net displacement, rather than a velocity kick [18], and section 5 concludes the discussion of electromagnetic memory's connections and consequence. 


\section{Conventions}

We will now summarize our conventions. In all three iterations of the symmetry/soft factor/memory triangle, computations are best performed in retarded and advanced coordinates. The flat Minkowski metric is:

$$
\begin{aligned}
d s^{2} & =-d u^{2}-2 d u d r+2 r^{2} \gamma_{z \bar{z}} d z d \bar{z} & & (u=t-r) \\
& =-d v^{2}+2 d v d r+2 r^{2} \gamma_{z \bar{z}} d z d \bar{z} & & (v=t+r)
\end{aligned}
$$

in retarded $(u)$ and advanced $(v)$ coordinates, where $\gamma_{z \bar{z}}=\frac{2}{(1+z \bar{z})^{2}}$ is the round metric on the $S^{2}$, with $(z, \bar{z})$ coordinates describing the stereographic projection of the Riemann sphere

$$
\hat{x}=\frac{1}{1+z \bar{z}}(z+\bar{z}, i(\bar{z}-z), 1-z \bar{z}) .
$$

The four-momentum of an on-shell massless particle can thus be parameterized by an energy $(\omega)$ and a direction on the $S^{2}$ :

$$
q^{\mu}=\omega(1, \hat{q})
$$

On a Penrose diagram, massive particles enter at past timelike infinity $i^{-}$and exit at future timelike infinity $i^{+}$, while massless particles enter at past null infinity $\mathcal{I}^{-}$and exit at future null infinity $\mathcal{I}^{+}$(see figure 1 ).

Thinking of quantities such as the asymptotic gauge fields or metric as living on the $\mathbb{R} \times S^{2}$ of future or past null infinity allows one to separate out the massless from the massive degrees of freedom. However, when computing quantities that live on null infinity, there should be a way to pull the physical observables into the bulk and make statements at large-but-finite $r$ and also for massive detectors (the generators along $\mathcal{I}$ are null).

In setting up a large- $r$ observing sphere, accelerating charges/masses sourcing the radiation are assumed to be at a small distance from the center of the sphere compared to the radius $\left|r_{s}\right| \ll|r|$. When comparing to Ward identities and other quantities described in terms of data - in particular zero modes — along null infinity [17], one should keep in mind that an integral along $v$ on past null infinity and then $u$ on future null infinity captures the incoming and outgoing radiation that would be seen by a large fixed- $r$ observer in the "radiation zone", integrating over all time $t$ (or any other of time coordinate $\{u, v, t\}$ at fixed large but finite $r$ when we are in Minkowski space). When one "integrates over all time" the relevant changes in the gauge field should start after an early enough $v$ and stop at some late enough $u$, so that a finite time integration can still capture the relevant observables.

If one thinks of the measurement sphere as extruding a cylinder $\left(\mathbb{R} \times S^{2}\right)$ in spacetime, all massless matter fluxes through the walls of the cylinder while massive matter goes through the endcaps (purple outline in figure 1). One must take this into account since states where the particles are moving with constant velocities at early and late times will eventually cross the sphere at some point, but a massive particle will never reach $\mathcal{I}^{+}$. The time interval starts and stops the clock when the massive particles are well within the sphere. Detectors sitting on the sphere in that interval still capture all of the radiation. 


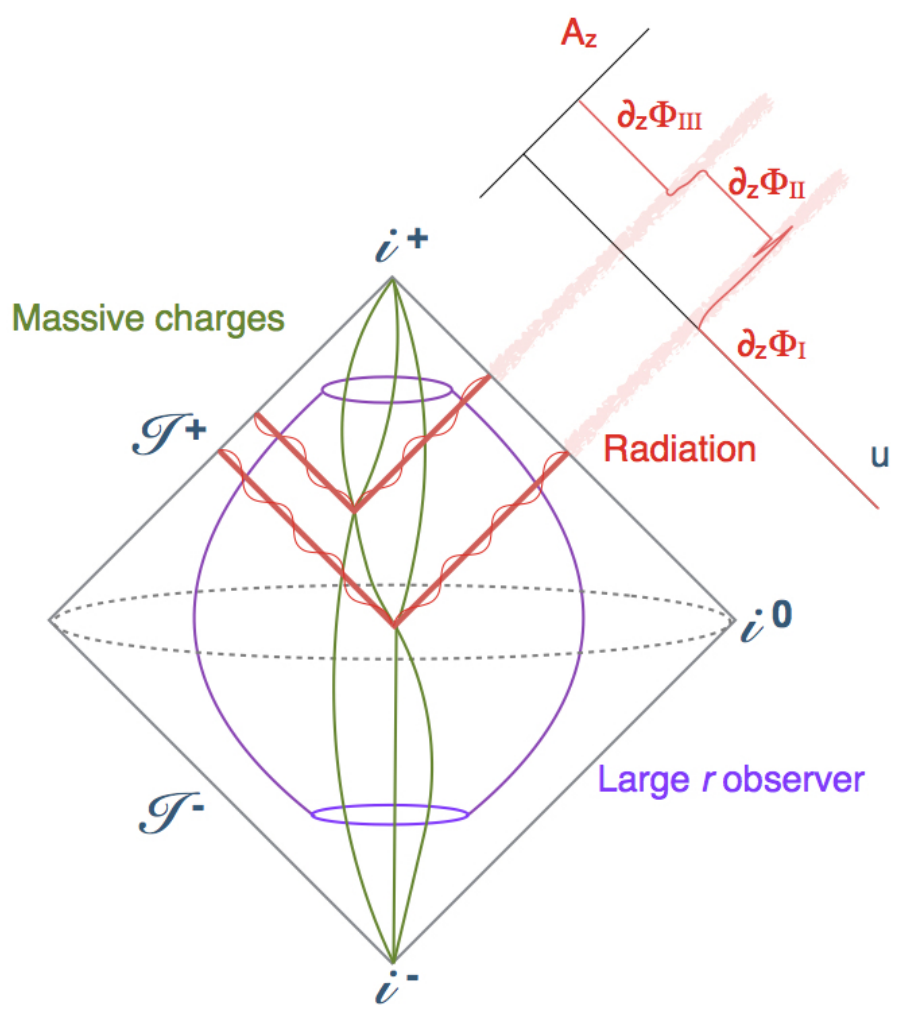

Figure 1. Radiation resulting from the acceleration of charges.

(Equivalently, one could restrict oneself to detectors at a large angular separation from where the particles enter or emerge to maintain the radiative $\frac{1}{r}$ power counting.) This is an intuitive picture for what is made precise by the boundary conditions we impose on the fields.

\section{Maxwell in the radiation zone}

\subsection{Boundary conditions}

There are two sets of boundary conditions relevant to discussing asymptotic symmetries and the electromagnetic memory effect. First, specifying the radial fall-off conditions on the electromagnetic fields allows one to solve for the radiation-zone solution to Maxwell's equations. Second, placing matching conditions on the gauge potential across spatial infinity $i^{0}$, and adding field strength boundary conditions at the temporal extremes of past and future null infinity, allows one to establish $\mathcal{S}$-matrix symmetries. There is more flexibility in the second step. Multiple methods can consistently give a "memory effect" with varying degrees of utility as an asymptotic $\mathcal{S}$-matrix symmetry.

The derivations of the relevant classical field equations in [17] and [18] are equivalent with respect to the first of these two steps. The fall-off conditions in Cartesian coordinates include: i) an $\mathcal{O}\left(\frac{1}{r^{2}}\right)$ radial electric field, ii) $\mathcal{O}\left(\frac{1}{r}\right)$ radiative fields, and iii) vanishing radial magnetic field (at each angle) at very early and very late times. In checking that this is 
consistent with the retarded radial coordinates of [17] one should keep in mind the factor of $r$ accompanying components tangential to the $S^{2}$ coming from the Jacobian $\partial_{z} x^{\mu}$.

For later use, let us make explicit our conventions for the gauge field components, following [17]. Here, one assumes the gauge field $\mathcal{A}_{\mu}(r, u, z, \bar{z})$ can be expanded in powers of $\mathcal{O}\left(\frac{1}{r}\right)$. In the retarded radial gauge choice of [17]:

$$
\begin{aligned}
\mathcal{A}_{r} & =0 \\
\left.\mathcal{A}_{u}\right|_{\mathcal{I}^{+}} & =0
\end{aligned}
$$

the radial fall-offs are $\mathcal{A}_{u}=\mathcal{O}\left(r^{-1}\right), \mathcal{A}_{z}=\mathcal{O}(1)$. We will define $A_{\mu}(u, z, \bar{z})$ as the leading coefficients of the $\frac{1}{r}$ expansion of $\mathcal{A}_{\mu}(r, u, z, \bar{z})$. Performing a radial expansion of $\mathcal{F}=d \mathcal{A}$ one has $\mathcal{F}_{u r}=\mathcal{O}\left(r^{-2}\right), \mathcal{F}_{z \bar{z}}=\mathcal{O}(1), \mathcal{F}_{u z}=\mathcal{O}(1)$. If we similarly defining $F_{\mu \nu}$ as the corresponding leading coefficients in the radial expansion of $\mathcal{F}_{\mu \nu}$, we get the following relations for the large $r$ limit of the field strength tensor [17]:

$$
\begin{aligned}
& F_{u r}=A_{u} \\
& F_{z \bar{z}}=\partial_{z} A_{\bar{z}}-\partial_{\bar{z}} A_{z} \\
& F_{u z}=\partial_{u} A_{z} .
\end{aligned}
$$

Note that $F_{u r}$ corresponds to the radial electric field $\left(A_{u}=-e^{2} r^{2} E_{r}\right), F_{z \bar{z}}$ to the radial magnetic field, and $F_{u z}$ to the radiative fields (tangent to the $S^{2}$ ). We will also impose $F_{z \bar{z}}=0$ at the boundaries of $\mathcal{I}^{+}\left(\operatorname{denoted} \mathcal{I}_{-}^{+}\right.$and $\left.\mathcal{I}_{+}^{+}\right)$.

The second step of boundary matching will be considered in section 3.4. The fundamental choice one confronts is whether to choose only retarded radiation solution or some other propagator to solve Maxwell's equations. The underlying question is whether to consider the charges taking part in a scattering process as transmitters or receivers. If one shoots a charged mass down an otherwise straight, rigid, frictionless wire with a kink in it (we can just as well smooth it out to a rounded elbow), then one can imagine that the mechanical forces causing the charge to accelerate as it rounds the bend will also cause it to emit radiation, making the retarded solution the best choice. On the other hand, one could look at the effects of incoming radiation on a set of charges. Explicit CPT symmetry ends up preferring a symmetric combination of incoming and outgoing radiation.

\subsection{Electromagnetic memory}

In this section, we specify a "memory effect" operator (3.8) for the U(1) case after a brief discussion of what we mean by memory observables in the first place - i.e. what criterion we use to distinguish the classical observable that we call a "memory". The term stems from the gravitational memory effect (see [1] for a review), where an array of test masses receive a finite nudge in position as a result of radiation. Given a scattering process, solving the linearized Einstein equations for the metric perturbation gives a net change in distance. Gravitational waves (e.g. from an inspiraling binary system) can themselves source such a perturbation in the metric. One often hears this referred to as the "nonlinear" Christodoulou effect; however, the same equations can be used to calculate the shift after including the gravitational contribution to the stress tensor (see the constraint equations in [2]). 
The essence of this process and its measurement is a "net effect", (i.e. it probes the zerofrequency limit of the gauge field sourcing the radiation). This picking out of zero-frequency modes comes from time integration. In gauge theory and gravity one can construct specific time integrated quantities determined by the same variables used to define |in $\rangle$ and |out $\rangle$ states, making it possible to connect them to $\mathcal{S}$-matrix Ward identities. Meanwhile, the fact that these $\mathcal{S}$-matrix related quantities pick out the zero-frequency modes of the corresponding gauge field motivates why they are connected to soft factors. The key ingredient to linking these phenomena is the ability to transition between position and momentum space.

The underlying principle behind the memory effect/soft theorem link is the connection between position and momentum space which can be made for massless modes as they approach null infinity. It relies on the saddle point approximation picking out $\hat{q} \cdot \hat{x}=1$ in the Fourier transformation of the massless field. Consider the mode expansion of the vector potential in the free Maxwell theory [17]:

$$
\mathcal{A}_{\mu}(x)=e \sum_{\alpha= \pm} \int \frac{d^{3} q}{(2 \pi)^{3}} \frac{1}{2 \omega_{q}}\left[\epsilon_{\mu}^{\alpha^{*}}(\vec{q}) a_{\alpha}(\vec{q}) e^{i q \cdot x}+\epsilon_{\mu}^{\alpha}(\vec{q}) a_{\alpha}(\vec{q})^{\dagger} e^{-i q \cdot x}\right] .
$$

In retarded coordinates the plane wave phase becomes:

$$
e^{i q \cdot x}=e^{-i \omega u-i \omega r(1-\hat{q} \cdot \hat{x})},
$$

and one sees that having the integral over on-shell momenta pick out the parallel direction comes from the order of limits, $r \rightarrow \infty$ first (i.e. before taking $|u|$ large). Moreover, the polarization choice $[16,17]$

$$
\begin{aligned}
& \epsilon^{+\mu}(\vec{q})=\frac{1}{\sqrt{2}}(\bar{w}, 1,-i,-\bar{w}), \\
& \epsilon^{-\mu}(\vec{q})=\frac{1}{\sqrt{2}}(w, 1, i,-w),
\end{aligned}
$$

convenient for evaluating soft factors, matches the gauge choice (3.1) to the relevant order in $\frac{1}{r}$. Then, for $\hat{q}$ parameterized by $w$ (analogous to (2.2)), one finds that in this collinear $\hat{q} \cdot \hat{x}=1$ limit

$$
\left.\epsilon_{z}^{-}(\vec{q})\right|_{\hat{q} \cdot \hat{x}=1}=\left.\partial_{z} x^{\mu} \epsilon_{\mu}^{-}(\vec{q})\right|_{\hat{q} \cdot \hat{x}=1}=\frac{\sqrt{2} r}{1+z \bar{z}},\left.\quad \epsilon_{z}^{+}(\vec{q})\right|_{\hat{q} \cdot \hat{x}=1}=\left.\partial_{z} x^{\mu} \epsilon_{\mu}^{+}(\vec{q})\right|_{\hat{q} \cdot \hat{x}=1}=0,
$$

and similarly for $\epsilon_{\bar{z}}^{ \pm}$using $\epsilon_{\mu}^{ \pm}=\left(\epsilon_{\mu}^{\mp}\right)^{*}$. One gets for the leading in $\frac{1}{r}$ radiative components [17]:

$$
A_{z}(u, z, \bar{z}) \equiv \lim _{r \rightarrow \infty} \partial_{z} x^{\mu} \mathcal{A}_{\mu}(x)=-\frac{i}{8 \pi^{2}} \frac{\sqrt{2} e}{1+z \bar{z}} \int_{0}^{\infty} d \omega\left[a_{+}(\omega \hat{x}) e^{-i \omega u}-a_{-}(\omega \hat{x})^{\dagger} e^{i \omega u}\right] .
$$

One sees from (3.7) that the operator

$$
\Delta A_{z} \equiv \int_{-\infty}^{\infty} d u \partial_{u} A_{z}(u, z, \bar{z})
$$


acting on an outgoing state picks out the mode $\lim _{\omega \rightarrow 0} \omega a_{+}(\omega \hat{x})$, extracting the residue of the $\frac{1}{\omega}$ Weinberg soft photon pole. By comparing to (3.2) we can identify this observable as the retarded-time integrated radiative electric field. As pointed out by [18], the electromagnetic analog of the gravitational memory effect amounts to the time integrated radiated electric field, so we can thus identify $\Delta A_{z}$ as a "memory effect" operator. (Note subleading terms are suppressed by $\mathcal{O}\left(\frac{1}{r}\right)$ in position space and $\mathcal{O}(\omega)$ for the soft photon, in momentum space.)

The saddle point has the following implication: the Weinberg soft factor in electromagnetism corresponds to the time integral of the radiated electric field where one replaces $\hat{q}$, describing the direction of the emitted soft photon, with the $\hat{x}$ for the position of the large- $r$ observer measuring the radiation. We will now see why this observable has the property of memory effects mentioned above regarding its dependence on the same data used to specify the $\mid$ in $\rangle$ and $\mid$ out $\rangle$ states for $\mathcal{S}$-matrix elements.

Consistent with [18] but in the notation of [17], the relevant Maxwell equation is

$$
\partial_{u} A_{u}=\partial_{u}\left(D^{z} A_{z}+D^{\bar{z}} A_{\bar{z}}\right)+e^{2} j_{u}
$$

where $D$ denotes a covariant derivative with respect to the unit $S^{2}$ and $j_{u}$ is the $\mathcal{O}\left(r^{-2}\right)$ term in the electric charge current. This is the constraint equation analogous to Gauss's law for future null infinity. Integrating along $u$, one gets at each angle,

$$
\Delta A_{u}=2 D^{z} \Delta A_{z}+e^{2} \int d u j_{u}
$$

where we have used $F_{z \bar{z}}=0$ at the boundaries of $\mathcal{I}^{+}$, as described after (3.2), which also implies that $\Delta A_{z}=\partial_{z} \phi$ for some function $\phi(z, \bar{z})$. Since we will be considering outgoing radiation when comparing to the retarded Liénard-Wiechert solution, we will be looking at insertions of our memory effect operator on outgoing states. Integrating along $u$ at fixed $r$ is equivalent to integrating for all times the radiation that reaches that detector, and for the outgoing case this measured radiation will be emerging from, rather than entering, the observing sphere. When all of the charges are massive, the $j_{u}$ term is zero, and one finds that the integrated gauge field is related to the change in the radial electric field. This is the Coulomb term. The key then is to look at the radial electric field for a constantly moving charge. We will next show that the Weinberg soft factor gives precisely the change in radial electric field $\Delta A_{u}$ for given initial and final configurations of boosted charges.

\subsection{Weinberg soft factor}

In this section, we use Weinberg's soft photon theorem to evaluate an insertion of our memory effect operator (3.8) on outgoing states in an $\mathcal{S}$-matrix element. The simplest way of seeing the connection between the Weinberg soft factor and the above electromagnetic memory effect is to make a few more assumptions about trajectories so one can evaluate $\Delta A_{u}$ for the Liénard-Wiechert solution and show that it is the same as the Weinberg soft factor with $\hat{q}$ for the soft photon replaced by $\hat{x}$ giving the location of the observer. 
First, consider the radial electric field of a boosted, but constantly moving charge for $\left|r_{s}\right| \ll|r|$ evaluated at $\vec{x}=r \hat{n}$ in terms of the position of the charge at the retarded time:

$$
E_{r}=\frac{Q}{4 \pi r^{2}} \frac{1}{\gamma^{2}(1-\vec{\beta} \cdot \hat{n})^{2}}
$$

Next, note that the electromagnetic soft factor contribution for a massive particle with momentum $p$ is:

$$
S_{p}^{(0) \pm}=e Q \frac{p \cdot \epsilon^{ \pm}}{p \cdot q}
$$

where explicitly

$$
p^{\mu}=m \gamma(1, \vec{\beta})
$$

and $\gamma$ with no indices refers to the Lorentz factor. This is the contribution from a single external particle to Weinberg's soft photon theorem:

$$
\left\langle\text { out }\left|a_{+}(\vec{q}) \mathcal{S}\right| \text { in }\right\rangle=S^{(0)+}\langle\text { out }|\mathcal{S}| \text { in }\rangle+\mathcal{O}\left(\omega^{0}\right)
$$

where the full soft factor is the signed sum of outgoing minus incoming charged particle contributions

$$
S^{(0)+}=\sum_{k \in \text { out }} S_{p_{k}}^{(0)+}-\sum_{k \in \text { in }} S_{p_{k}}^{(0)+}
$$

Considering outgoing radiation emitted during scattering, (3.7) and (3.14) give:

$$
\lim _{\omega \rightarrow 0} \frac{\left\langle\text { out }\left|\Delta A_{z} \mathcal{S}\right| \text { in }\right\rangle}{\langle\text { out }|\mathcal{S}| \text { in }\rangle}=-\frac{e}{4 \pi} \hat{\epsilon}_{z}^{*+} \omega S^{(0)+},
$$

where $\omega S^{(0)+}$ is $\mathcal{O}\left(\omega^{0}\right)$. Using (2.3) with $\hat{q}=\hat{n}$, we evaluate:

$$
-\frac{e}{4 \pi} \lim _{\omega \rightarrow 0} \omega\left[D^{z} \hat{\epsilon}_{z}^{*+} S_{p}^{(0)+}+D^{\bar{z}} \hat{\epsilon}_{\bar{z}}^{*-} S_{p}^{(0)-}\right]=-e^{2} \frac{Q}{4 \pi} \frac{1}{\gamma^{2}(1-\vec{\beta} \cdot \hat{n})^{2}},
$$

where $\hat{\epsilon}$ is the $r$-stripped polarization tensor in retarded radial coordinates (3.5). This is consistent with the expectation value interpretation of [1] if we compare the operator insertion (3.16) to (3.10) evaluated for the Liénard-Wiechert solution. By additionally assuming that the acceleration occurs over a small window during which the charges do not appreciably move from the center of the observing sphere, one can evaluate $\Delta A_{u}$ by subtracting the initial from the final $A_{u}$ of a superposition of constantly moving charges near the same value of $u$. This connects the single particle contribution to the soft factor to the radial electric field of the asymptotic configuration. Using $A_{u}=-e^{2} r^{2} E_{r}$, comparing the righthand side of (3.17) with (3.11), and summing up the contributions from each scatterer, we see that (3.17) and (3.16) exhibit the relation between $\Delta A_{u}$ and $\Delta A_{z}$ in (3.10).

Having early and late asymptotic states with constant on-shell velocities implies this $\Delta A_{u}$ corresponds to the electromagnetic soft factor. Consistency of Maxwell's equations at 
$\mathcal{I}^{+}$given a scattering process with no charges exiting $\mathcal{I}^{+}$, demands the outgoing radiation solution have a net impulse corresponding to the soft factor. ${ }^{1}$

\subsection{Large U(1) symmetry}

By 'large gauge symmetries' we are referring to a class of pure gauge transformations that act non-trivially on the boundary data. In particular, for certain large- $r$ falloffs one finds a symplectic paring between these modes and the physical memory modes [17], indicating that despite their pure gauge nature, they must be included in the phase space. In this section, we review the derivation of the asymptotic U(1) gauge symmetry found in [17] and discuss the second step in setting the boundary conditions for an $\mathcal{S}$-matrix symmetry.

As a primer, let us take a moment to consider how a residual large gauge symmetry can be seen as necessary for self consistency of the theory with radiation along $\mathcal{I}^{+}$. Consider the plot in the upper righthand corner of in figure 1. One can look at the gauge field at a particular angle on the $S^{2}$ as a function of $u$. The first round of boundary conditions (see text after (3.2) and (3.10)) results in the electromagnetic memory depending on a "pure gauge" function, i.e. $\Delta A_{z}=\partial_{z} \phi(z, \bar{z})$. Consider situations where the durations over which accelerations emitting radiation occur have compact support along $u$. Then separate out the intervals between scattering processes. This follows naturally from assuming one can isolate a single interaction. One should be able to measure the radiation over the time interval relevant to a particular process and extract information that does not depend on later processes. As such, one can imagine intervals of "pure gauge" between each such segment for well-separated events. Indeed, in the Light-Shell Effective Theory (LSET) solutions for massless scattering considered by [20], consistency with the soft factor comes from a step function profile in the radiation (on the $u=0$ shell propagating from an interaction at the spacetime origin).

t null infinity, the classical vacuum "picks out" a pure gauge $A_{z}$ configuration that is constant in $u$. If we consider a scattering process in which the $A_{z}$ background agrees with this profile at early retarded times, and then decide perform a large gauge transformation to "set" $A_{z}$ at early times to zero, one finds that Weinberg's soft theorem implies that at late times we will generically not have $\phi=0$. Moreover, given the picture of well-separated events, between any two wavefronts of radiation (i.e. in any of the three regions in figure 1),

\footnotetext{
${ }^{1}$ As a side note, the same analysis can be applied to the leading Weinberg pole in the gravity case. There the analog of the radial electric field is the boosted Bondi mass $m_{\mathrm{B}}$ in Bondi gauge. For massive scattering with no flux through $\mathcal{I}^{+}$the linearized constraint equation and soft factor/expectation value interpretation give:

$$
\Delta m_{\mathrm{B}}=\frac{1}{4}\left[D^{z} D^{z} \Delta C_{z z}+D^{\bar{z}} D^{\bar{z}} \Delta C_{\bar{z} \bar{z}}\right], \quad \Delta C_{z z}=-\frac{\kappa}{4 \pi} \hat{\epsilon}_{z z}^{*+} S^{(0)+}
$$

where the last relation is shorthand for the analog of (3.16) in light of the expectation value-like behavior observed here and in [1]. This is consistent with the analog of (3.17):

$$
-\frac{\kappa}{4 \pi} \lim _{\omega \rightarrow 0} \omega\left[D^{z} D^{z} \hat{\epsilon}_{z z}^{*+} S_{p}^{(0)+}+D^{\bar{z}} D^{\bar{z}} \hat{\epsilon}_{\bar{z} \bar{z}}^{*-} S_{p}^{(0)-}\right]=\frac{4 G m}{\gamma^{3}(1-\vec{\beta} \cdot \hat{n})^{3}}=4 m_{\mathrm{B}}(\vec{\beta}),
$$

where the second equality can be compared with $[4,5]$ for a boosted mass, and the single particle soft factor contribution is now $S_{p}^{(0) \pm}=\frac{\kappa}{2} \frac{\left(p \cdot \epsilon^{ \pm}\right)^{2}}{p \cdot q}$ with $\kappa=\sqrt{32 \pi G}$.
} 
we should be able to "reset" our baseline - i.e. perform a large gauge transformation to set $A_{z}=0$ over that no-radiation interval (which won't effect the physical radiation pulses but will change us to $\phi=$ const. in the intervals between them). Thus, while one zero mode corresponds to a step, it must naturally be accompanied by an overall shift at each angle which corresponds to the resetting. The gauge transformation necessary for such a resetting is the negative of the domain-wall like transition effected by a pulse of radiation hitting the detector between retarded times $u_{i}$ and $u_{f}$, which can be computed analogously to $(3.7)$ of $[1]$ :

$$
\Delta \phi(z, \bar{z})=\frac{1}{4 \pi} \int d^{2} z^{\prime} \gamma_{z^{\prime} \bar{z}^{\prime}} \log \left|z-z^{\prime}\right|^{2}\left[\Delta A_{u}-e^{2} \int_{u_{i}}^{u_{f}} d u j_{u}\right]
$$

up to a constant function in the kernel of $\partial_{z}$ and $\partial_{\bar{z}}$ (taking into account the reality of $A_{\mu}$ ). Here we have used (3.10), the condition on the late time behavior of $F_{z \bar{z}}$ to write $\Delta A_{z}=\partial_{z} \Delta \phi$, and $\partial_{z} \partial_{\bar{z}} \log \left|z-z^{\prime}\right|^{2}=2 \pi \delta^{(2)}\left(z-z^{\prime}\right)$.

Note that one can heuristically see how the presence of the Weinberg pole corresponds to a step from the fact that the Fourier transformation of a step function is a pole. Meanwhile, the Fourier transformation of a constant is a delta function, so this overall shift can be added in by hand to the standard Fourier transform modes [19] as a strictly zero frequency extension of the phase space. The fact that any classical vacuum spontaneously breaks the symmetry in choice of $A_{z}$ is the origin of the Goldstone mode interpretation of $\phi$.

Now to address the second step in boundary matching. Among the intriguing aspects of the $\mathcal{S}$-matrix approach of [17] is the way in which the Ward identity motivates a bracket between these two zero modes by considering the charge generating the large U(1) symmetry. The equations of motion (3.10) and the analogous one on $\mathcal{I}^{-}$are treated as operator statements when inserted into the $\mathcal{S}$-matrix. In constructing a Ward identity of the form

$$
\left\langle\text { out }\left|\left(Q_{\epsilon}^{+} \mathcal{S}-\mathcal{S} Q_{\epsilon}^{-}\right)\right| \text {in }\right\rangle=0
$$

boundary conditions setting $A_{u}=0$ at $\mathcal{I}_{+}^{+}$and $\mathcal{I}_{-}^{-}$, followed by antipodally matching $A_{z}$ at $\mathcal{I}_{-}^{+}$and $\mathcal{I}_{+}^{-}$across $i^{0}$ allow one to relate the current to the soft factor. This is a particular feature of massless scattering, where all the charges enter $\mathcal{I}^{-}$and exit $\mathcal{I}^{+}$and one can cancel the $\Delta A_{u}$ term between incoming and outgoing scatterers. (I.e. the massless soft factor obeys a differential equation that localizes on the $S^{2}$, just as the massless current does.)

The required mix of incoming and outgoing radiation comes from the way in which the current, as a generator of gauge transformations on the matter fields, only acts on the outgoing or incoming particles for $j_{u}$ or $j_{v}$, respectively, whereas an outgoing soft photon attaches to both incoming and outgoing legs (ditto for an incoming soft photon). As such, a factor of $\frac{1}{2}$ arises from averaging the incoming and outgoing radiation solutions to match the combined current contribution that counts incoming and outgoing particles only once each.

As a final note, we point out a connection to the $\phi=\phi_{m>0}+\phi_{m=0}$ splitting by [18] into components sourced by $\Delta A_{u}$ from massive charges and $e^{2} \int d u j_{u}$ from the massless current. 
The radiation response due to the massive charges results from a change in their kinematic distributions, whereas the response from the massless charges accounts for them exiting the sphere. Since the analysis of [18] considers only the solution near $\mathcal{I}^{+}$, one should use crossing symmetry to consider a neutral incoming state to connect with the analysis of [17]. (For the purpose of visualizing radiation arising from prescribed accelerations of scattered charges, one can imagine superimposing the non-radiating solution of an oppositely charged particle moving unperturbed through spacetime parallel to each incoming particle that scatters. While there is no incoming charge, an oppositely charged particle leaving at the antipodal angle maintains the outgoing-minus-incoming structure of the original soft factor). Between the results shown here and in [17], the soft factor is consistent with measuring the massless and massive contributions to the memory effect, independently. This separation provides insight into extending the [17] formalism to the massive case.

\section{Measurement}

Now that we have circuited the triad of connections relevant to the electromagnetic memory effect, let's consider a compact setup that could measure it. One wants a way to extract just the zero-mode effect, and we would like to prescribe a way of measuring this time integrated electric field that entails setting up, waiting for, and then making a final measurement. The proposition of [18] was to connect the time integrated electric field to a net velocity kick. Explicitly, a test charge obeying $\vec{F}=m \vec{a}=Q \vec{E}_{\text {rad }}$ has an acceleration proportional to $\frac{Q}{m}$ times the radiated electric field (which one should keep in mind is a $\frac{1}{r}$ effect, and the resultingly small velocity of the test charge will suppress the magnetic contribution to the force). If the pulse occurs over a short enough period of time that the test charge remains localized on the sphere, then it receives a net kick in its velocity $\Delta \vec{v}=\int d t \vec{a}$.

If we prefer to keep the test charge localized rather than letting it fly off at some velocity that would need to be measured (or, if restricted to the sphere, letting it move enough that a path integral of the tangential force would be required), we instead can imagine that at the location of where we want to measure the effect, we have a charged bead suspended in a viscous fluid. Rather than going too deep into how to realistically separate the scales of the interactions which govern the viscous forces between the bead and the fluid and the scattering-sourced radiation we want to measure, we can imagine an idealized situation where the viscous force dominates and any response to a driving force is proportional to the velocity (i.e. heavily damped rather than inertial). With a drag force at low Reynolds number of $\vec{F}_{D}=-\sigma \vec{v}$ for some positive constant $\sigma$, which dominates and balances the driving force from the radiated electric field, one finds:

$$
\int d t Q \vec{E}_{\mathrm{rad}}=\int d t \sigma \vec{v}=\sigma \Delta \vec{x}
$$

in this limit, so that the electromagnetic memory is turned into a net displacement (like in the gravitational memory case) rather than a velocity kick. To distinguish this effect, the relevant scattering process would need to induce a $\Delta \vec{x}$ larger than the expected drift of the test charge during the integration time, due to Brownian motion. 
Now that we have some sense of how to measure the electromagnetic memory, it is interesting to reflect on how this observable is distinct from other radiation measurements. What intrigues us about the electromagnetic memory is its universal dependence on the incoming and outgoing asymptotic states of the charged particles, while being linear in the electric field. As [18] points out, a typical photon detector would measure the electromagnetic energy flux, which is quadratic in the field strength. Given the initial and final momenta, Maxwell's equations constrain the net time integrated radiated electric field at any given angle. This corresponds to the electromagnetic memory. However, one can imagine distributing this radiation over a very slow ramp. If we tune down the rate at which charges accelerate, we can make the power flux arbitrarily small, while keeping the same value of the net integrated field because the ramp integrates to the same end point but takes longer to get there. To keep the position of the accelerating charges near the origin $\left|r_{s}\right| \ll|r|$ during this ramp, we can simultaneously consider detectors that are further away to maintain the order of limits consistent with $r \rightarrow \infty$ first. The upshot is that the feature (dependence on initial and final boundary conditions for charge velocities rather than acceleration profile) of the electromagnetic memory effect that makes it hidden from typical detectors is also what makes it universal. As such, there is a sense in which the low energy observables captured by the memory effect are distinct from the quantities typically measured.

\section{Discussion}

In summary, we have seen that the connection between asymptotic symmetries, soft factors, and memory effects extends naturally to the U(1) case and rounds out the interpretation of any individual link or vertex in this triad. Memory effects pick out zero-mode classical observables. Meanwhile, the position space interpretation of soft factors connects large distances with low frequency radiation in the same direction. In this manner, soft factors can both: i) lead to Ward identities that validate the quantum versions of these symmetries, and ii) give the expectation value of classical radiation measurements. Furthermore, the ability to superimpose classical radiation solutions corresponding to the memory effect for separate scattering processes, combined with the freedom to reset the gauge field between pulses of radiation when performing calculations, illustrates from a semi-classical perspective how the presence of "pure gauge" zero modes are essential for self consistency and should be included in the extended phase space.

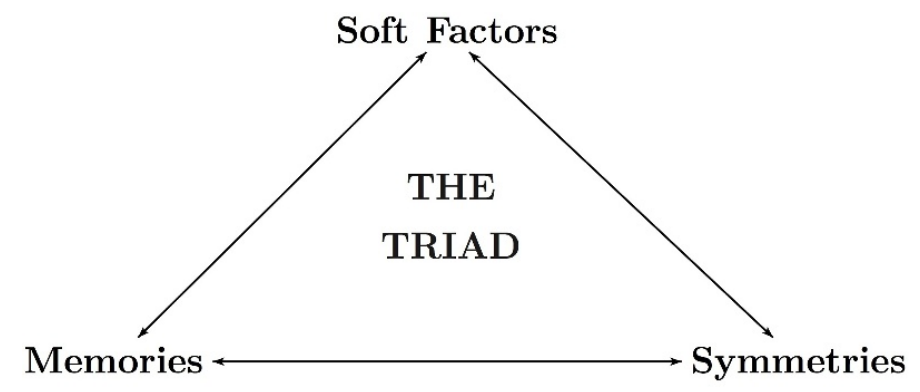




\section{Acknowledgments}

Many thanks to J. Barandes and A. Zhiboedov. Thank you to G. Compère, M. Schwartz, and A. Strominger for useful questions. This work coalesced while preparing for Harvard String Family and MIT LHC/BSM Journal Club talks. I am grateful to the LHC/BSM Journal Club and L. Susskind for convincing me to arXiv my notes. This work was supported in part by the Fundamental Laws Initiative at Harvard, the Smith Family Foundation, the National Science Foundation, and the Hertz Foundation.

Open Access. This article is distributed under the terms of the Creative Commons Attribution License (CC-BY 4.0), which permits any use, distribution and reproduction in any medium, provided the original author(s) and source are credited.

\section{References}

[1] A. Strominger and A. Zhiboedov, Gravitational Memory, BMS Supertranslations and Soft Theorems, JHEP 01 (2016) 086 [arXiv:1411.5745] [INSPIRE].

[2] S. Pasterski, A. Strominger and A. Zhiboedov, New Gravitational Memories, JHEP 12 (2016) 053 [arXiv: 1502.06120] [INSPIRE].

[3] S. Weinberg, Infrared photons and gravitons, Phys. Rev. 140 (1965) B516 [InSPIRE].

[4] H. Bondi, M.G.J. van der Burg and A.W.K. Metzner, Gravitational waves in general relativity. 7. Waves from axisymmetric isolated systems, Proc. Roy. Soc. Lond. A 269 (1962) 21 [INSPIRE].

[5] R.K. Sachs, Gravitational waves in general relativity. 8. Waves in asymptotically flat space-times, Proc. Roy. Soc. Lond. A 270 (1962) 103 [InSPIRE].

[6] A. Strominger, On BMS Invariance of Gravitational Scattering, JHEP 07 (2014) 152 [arXiv:1312.2229] [INSPIRE].

[7] T. He, V. Lysov, P. Mitra and A. Strominger, BMS supertranslations and Weinberg's soft graviton theorem, JHEP 05 (2015) 151 [arXiv:1401.7026] [INSPIRE].

[8] Y B. Zeldovich and A.G. Polnarev, Radiation of gravitational waves by a cluster of superdense stars, Sov. Astron. 18 (1974) 17.

[9] V.B. Braginsky and K.S. Thorne, Gravitational-wave bursts with memory and experimental prospects, Nature 327 (1987) 123.

[10] D. Christodoulou, Nonlinear nature of gravitation and gravitational wave experiments, Phys. Rev. Lett. 67 (1991) 1486 [INSPIRE].

[11] T. Banks, A Critique of pure string theory: Heterodox opinions of diverse dimensions, hep-th/0306074 [INSPIRE].

[12] G. Barnich and C. Troessaert, Symmetries of asymptotically flat 4 dimensional spacetimes at null infinity revisited, Phys. Rev. Lett. 105 (2010) 111103 [arXiv:0909.2617] [INSPIRE].

[13] G. Barnich and C. Troessaert, Supertranslations call for superrotations, PoS (CNCFG2010) 010 [arXiv: 1102.4632] [INSPIRE].

[14] G. Barnich and C. Troessaert, BMS charge algebra, JHEP 12 (2011) 105 [arXiv:1106.0213] [INSPIRE]. 
[15] F. Cachazo and A. Strominger, Evidence for a New Soft Graviton Theorem, arXiv: 1404.4091 [INSPIRE].

[16] D. Kapec, V. Lysov, S. Pasterski and A. Strominger, Semiclassical Virasoro symmetry of the quantum gravity $\mathcal{S}$-matrix, JHEP 08 (2014) 058 [arXiv: 1406.3312] [INSPIRE].

[17] T. He, P. Mitra, A.P. Porfyriadis and A. Strominger, New Symmetries of Massless QED, JHEP 10 (2014) 112 [arXiv:1407.3789] [INSPIRE].

[18] L. Bieri and D. Garfinkle, An electromagnetic analogue of gravitational wave memory, Class. Quant. Grav. 30 (2013) 195009 [arXiv:1307.5098] [INSPIRE].

[19] S. Pasterski, Classical Interpretation of the Weinberg Soft Factor, Bowker, New Providence The Bahamas (2014) [ISBN: 978-0-9863685-8-5].

[20] H. Georgi, G. Kestin and A. Sajjad, Towards an Effective Field Theory on the Light-Shell, JHEP 03 (2016) 137 [arXiv: 1401.7667] [INSPIRE]. 